\title{
Leveraging information and collaboration to cure disease
}

\author{
David Fajgenbaum \\ MD, MBA, MSc., Assistant Professor of Medicine, Division of Translational Medicine \& Human \\ Genetics, Perelman School of Medicine, University of Pennsylvania, 3400 Spruce Street, Silverstein 5, \\ S05094, Philadelphia, PA 19104, USA \\ Tel.: 215-614-0936; E-mail: davidfa@mail.med.upenn.edu
}

\begin{abstract}
Despite many medical breakthroughs in the last century, many diseases are still devastating and incurable. One such disease is idiopathic multicentric Castleman disease (iMCD). iMCD is a deadly hematologic illness where the immune system attacks and shuts down the body's vital organs for an unknown cause. Several barriers related to funding, classification systems, (lack of) diagnostic criteria, studies with small sample sizes, and collaboration slowed progress for iMCD. The traditional model for how to advance biomedical research also had many shortcomings. The Castleman Disease Collaborative Network (CDCN) was created in 2012 to take an innovative, "collaborative network approach" to accelerate research and treatment for Castleman disease. The $\mathrm{CDCN}$ has facilitated collaboration, leveraged available information, coordinated research, and engaged key stakeholders to make paradigm-shifting progress. The vital steps, key progress, and lessons learned are shared in this paper.

Keywords: Castleman disease, rare disease, orphan disease, collaboration, research
\end{abstract}

\section{Introduction}

Modern biomedical research techniques have enabled unprecedented advances in our understanding and treatment of disease. However, many hurdles exist that impede the pace of progress. Despite the many medical breakthroughs over the last century, many devastating and incurable diseases remain. One disease that has evaded modern research breakthroughs and treatment is idiopathic multicentric Castleman disease (iMCD). iMCD is a deadly hematologic illness where the immune system attacks and shuts down the body's vital organs for an unknown cause. I (David Fajgenbaum) first learned about this disease when I was a third-year medical student. Unlike other diseases, I didn't read about it in a textbook or learn about it in a lecture. It nearly killed me in 2010 - for the first time. I have considered that moment to be the start of my "overtime" and have dedicated every second since then to trying to cure iMCD by facilitating collaboration, leveraging available information, coordinating research, and engaging key stakeholders. Herein, I will share aspects of my personal journey, hurdles that I observed for Castleman disease and biomedical research, solutions that I developed for Castleman disease and biomedical research, progress that we have made, and lessons learned.

\section{My personal journey}

I grew up in Raleigh, NC laser-focused on one day playing Division I college football. Georgetown University recruited me to play quarterback, and I enrolled in 2003. Shortly after arriving on campus, 
I learned that my mother had been diagnosed with terminal brain cancer. I struggled over the next fifteen months while my mother bravely battled cancer. Just before she passed away in 2004, I assured her that I would be ok and that I would start an organization in her memory to support grieving college students. I called the group AMF, which stood for Ailing Mothers \& Fathers and my mother's initials. I also decided that I wanted to become an oncologist to treat cancer patients like my mother. In my third year of medical school, I became suddenly ill with flu-like symptoms, abdominal pain, and fluid accumulation. I was hospitalized in critical condition with liver, kidney, and bone marrow failure. A retinal hemorrhage made me blind in my left eye, and I gained over 50 pounds of fluid. After nearly dying twice, I was eventually diagnosed with iMCD and chemotherapy was initiated. Just a month later, I relapsed again and required a third month-long hospitalization. This time, I was given a combination of seven chemotherapies that obliterated my immune system and put my disease in remission. After nearly five months hospitalized, I was discharged and returned to medical school a few months later. I knew that iMCD research was being done at a few centers around the world, and I trusted that someone else could unravel the mysteries of iMCD. I did not get involved in iMCD research immediately, but I did write up a case report on myself where I was the case and the first author [2]. I relapsed 15 months later and realized that I could no longer hope that someone else would answer the unknowns of iMCD. I would need to dedicate the rest of my life to studying it.

\section{Idiopathic multicentric Castleman disease}

iMCD is one of three subtypes of Castleman disease, which is diagnosed in approximately six thousand to seven thousand individuals per year in the USA - approximately the same incidence as Amyotrophic Lateral Sclerosis (ALS) [7]. iMCD causes flu-like symptoms, declines in blood counts, fluid accumulation, enlargement of liver, spleen, and lymph nodes, and multiple organ system dysfunction. There is a three-fold increased prevalence of cancer among patients with iMCD compared to agematched controls [6]. Thirty-five percent of patients die within five years of diagnosis, which is a worse prognosis than lymphoma, breast cancer, and prostate cancer [1]. Siltuximab, the only FDAapproved treatment for iMCD, is highly-effective, but only in approximately one-third of patients [8]. Optimal treatment for the remaining two-thirds of patients is unknown due to the many unanswered questions about iMCD pathogenesis, including: (1) why does the immune system become activated and attack the vital organs? (2) which immune cell type is responsible for initiating the attack? (3) what signaling pathways are dysregulated in these patients? and (4) what treatments are effective for this disease?

\section{State of iMCD research in 2012}

First, I performed an analysis of the state of iMCD research in 2012. I found that researchers were not collaborating. The literature was disorganized and confusing as authors used different classification and terminology systems to describe the subtypes of Castleman disease. No one knew what triggered $\mathrm{iMCD}$, the immune cells involved, or the dysregulated signaling pathways. The framework for thinking about the disease didn't make sense. There were no diagnostic criteria. Siltuximab had shown promising results in clinical trials, but it was not yet approved. No advocacy organization or NIH-funded projects were in place. 
Next, I investigated the typical routes that research foundations take to advance biomedical research. I found that organizations typically raise funding, invite researchers to apply to use the funding, and then award the funding to the best applications. Thus, research does not occur as part of a coordinated, overarching strategy. It occurs in a somewhat random fashion - foundations/patients/loved ones must hope that the right researcher with the right skillset will have the right idea for the right project at the right time. Furthermore, it fragments the community to compete instead of collaborating.

I decided that I would create a research foundation, the Castleman Disease Collaborative Network $(\mathrm{CDCN})$, to spearhead a new approach to research that was more strategic, efficient, and collaborative.

\section{The collaborative network approach}

I established a new approach to research called the "Collaborative Network Approach" [3]. Rather than raising money and inviting researchers to apply for it, we: (1) identified and built a community of researchers and a community of patients, (2) leveraged the communities to contribute research ideas and prioritize them into an International Research Agenda, (3) execute the research agenda through targeted fundraising, procuring the necessary patient samples, and recruiting the top international experts to perform the priority projects, (4) translate findings to identify drugs already approved for other conditions that may be effective for iMCD, and (5) track the effectiveness of these new and old treatments through a registry. In parallel, we collect and share samples and data for research, connect and support patients, and coordinate the execution of our International Research Agenda with the support of medical students, professionals, patients, and families.

We also utilize novel technologies to advance research. To facilitate collaboration and dissemination of knowledge among physicians and researchers, we established a private discussion board for sharing of ideas and publications in real-time. We also host a portal for patients to connect with one another. To promote innovative research studies, we utilize crowdsourcing to identify high-impact research projects. We use a platform (www.codigital.com) to directly collect research ideas from patients and physicians, who can propose ideas and up-vote, down-vote, or edit others' ideas. We also curate comments on the physician and patient discussion boards to identify themes and trends that may warrant further research. To ensure that our research funds are spent as efficiently as possible, we recruit the top experts to perform the research studies proposed through crowdsourcing and prioritized by our Scientific Advisory Board. We also connect physicians and researchers around the world to share samples and data for specific research studies. We have built an international natural history registry to collect data on the effectiveness of off-label treatments for iMCD.

\section{Progress over five years}

We have made tremendous progress over the last five years. We established a unifying terminology system and new model of pathogenesis based on a review of published data and communications with experts [5]. We performed a systematic literature review to characterize clinical features, associated diseases, risk of malignancy, and effective treatments for iMCD [4]. Then, we combined the data from the systematic literature review with additional cases to establish the first-ever evidence-based, expertconsensus international diagnostic criteria for iMCD [4]. In parallel, we launched a pathogen discovery study, genomic sequencing of germline DNA and lymph node tissue DNA, flow cytometry, RNA sequencing, serum proteomics, international natural history registry, and biobank. 
Today, there are over four hundred physicians and researchers connected through the CDCN, which is led by a thirty-two-member Scientific Advisory Board from eight countries on five continents. We have coordinated the five largest ever Castleman disease meetings, successfully lobbied for a unique ICD-10 code (see: https://www.cdc.gov/nchs/icd/icd10cm.htm) for Castleman disease, and identified and described a new clinical subtype of iMCD. Siltuximab became the first FDA-approved treatment for iMCD in 2014. We have also connected over six thousand iMCD patients and loved ones virtually and supported patients through education about iMCD, referrals to leading experts, and connections with other patients.

\section{Lessons learned \& next steps}

Leaders in biomedical information services must question the status quo and come up with innovative solutions to serious challenges that exist in biomedical research. However, nothing can be done in isolation. We must all work together and collaborate. Leveraging the crowd is an important tool to identify novel and high-impact solutions, but it is essential to have an expert panel that can prioritize the crowdsourced ideas. We must come up with new ways to share data and samples more quickly. The timeline from data generation to publication to dissemination is too long. Biomedical research is iterative, and we need to shorten the time for each cycle of iteration. Many diseases do not have effective treatments, so off-label drugs are used. We must come up with systems to quantify the effectiveness of these treatments in real-time to inform treatment of future patients.

Castleman disease is often called "Castleman's disease" despite the National Library of Medicine's best attempts to remove apostrophe "s" from the medical literature and ontologies. Unfortunately, both "Castleman disease" and "Castleman's disease" are used in the medical literature. When someone searches PubMed for one of the spellings, then only papers with that spelling are included in the results - meaning approximately one-half of all papers are left out of the results for any search by a physician or researcher into Castleman disease. This can have major consequences as new diagnostic criteria or data on treatment options may not appear for a physician needing this information. I asked the audience to help me with this issue, which I hope that we can solve. I encourage anyone who is interested in helping with this or any of our other efforts to cure Castleman disease to reach out to me at davidfa@mail.med.upenn.edu or visitwww.CDCN.org.

\section{Acknowledgements}

I wish to thank my wife, Caitlin, for her unwavering support, and colleagues at the CDCN, especially Jason, Dustin, Mary, Helen, Patty, and Mileva for their efforts to cure Castleman disease.

\section{About the author}

Dr. Fajgenbaum is Assistant Professor of Medicine at UPenn and co-founder of the Castleman Disease Collaborative Network (CDCN). He is also a patient battling a rare and deadly disease - idiopathic multicentric Castleman disease. Fajgenbaum and the CDCN's research has changed the way Castleman disease is researched and treated. 


\section{References}

[1] A. Dispenzieri, J.O. Armitage, M.J. Loe, S.M. Geyer, J. Allred and J.K. Camoriano, The clinical spectrum of Castleman's disease, American Journal of Hematology 87(11) (2012), 997-1002. doi:10.1002/ajh.23291.

[2] D. Fajgenbaum, M. Rosenbach, F. van Rhee, A. Nasir and J. Reutter, Eruptive cherry hemangiomatosis associated with multicentric Castleman disease: A case report and diagnostic clue, JAMA Dermatology 149(2) (2013), 204-208. doi:10. 1001/jamadermatol.2013.1552.

[3] D.C. Fajgenbaum, J.R. Ruth, D. Kelleher and A.H. Rubenstein, The collaborative network approach: A new framework to accelerate Castleman's disease and other rare disease research, The Lancet Haematology 3(4) (2016), e150-e152.

[4] D.C. Fajgenbaum, T.S. Uldrick, A. Bagg, D. Frank, D. Wu, G. Srkalovic et al., International, evidence-based consensus diagnostic criteria for HHV-8-negative/idiopathic multicentric Castleman disease, Blood 129(12) (2017), 1646-1657. doi:10. 1182/blood-2016-10-746933.

[5] D.C. Fajgenbaum, F. van Rhee and C.S. Nabel, HHV-8-negative, idiopathic multicentric Castleman disease: Novel insights into biology, pathogenesis, and therapy, Blood 123(19) (2014), 2924-2933. doi:10.1182/blood-2013-12-545087.

[6] A.Y. Liu, C.S. Nabel, B.S. Finkelman, J.R. Ruth, R. Kurzrock, F. van Rhee et al., Idiopathic multicentric Castleman's disease: A systematic literature review, The Lancet Haematology 3(4) (2016), e163-e175.

[7] N. Munshi, M. Mehra, H. van de Velde, A. Desai, R. Potluri and J. Vermeulen, Use of a claims database to characterize and estimate the incidence rate for Castleman disease, Leukemia \& Lymphoma 56(5) (2015), 1252-1260. doi:10.3109/ 10428194.2014.953145.

[8] F. van Rhee, R.S. Wong, N. Munshi, J.-F. Rossi, X.-Y. Ke, A. Fosså et al., Siltuximab for multicentric Castleman's disease: A randomised, double-blind, placebo-controlled trial, The Lancet Oncology 15(9) (2014), 966-974. doi:10.1016/S14702045(14)70319-5. 